\title{
Erratum
}

\section{Correction to Kawashima, Kawamoto, Shiraga, and Kawano (2020)}

In the article "Is suicide beautiful? Suicide acceptance and related factors in Japan" by D. Kawashima, S. Kawamoto, K. Shiraga, and K. Kawano (Crisis, 41(2), 114-120, https:// doi.org/10.1027/0227-5910/a000612) there were errors in the fourth paragraph of the results section and the second paragraph of the discussion section.

The fourth paragraph of the results section reads like this:

In addition, unemployed participants scored higher than full-time workers on both the beautification $(p=.001$, $d=.19)$ and justification subscales $(p=.005, d=.17$ ).

The corrected text should read as follows (correction in bold):

In addition, unemployed participants scored lower than full-time workers on both the beautification $(p=.001$, $d=.19)$ and justification subscales $(p=.005, d=.17)$.
The second sentence in the second paragraph of the discussion section should read as follows (correction in bold):

We observed that male and distressed participants found suicide more acceptable, perhaps because being male and being distressed are risk factors for suicide (Hawton \& van Heeringen, 2009).

The authors regret any inconvenience or confusion the errors may have caused.

\section{Reference}

Kawashima, D., Kawamoto, S., Shiraga, K., \& Kawano, K. (2020). Is suicide beautiful? Suicide acceptance and related factors in Japan. Crisis, 41(2), 114-120. https://doi.org/10.1027/0227-5910/a000612

Published online June 29, 2021 